University of Wollongong

Research Online

Faculty of Informatics - Papers (Archive)

Faculty of Engineering and Information

Sciences

10-4-2006

\title{
Electric field-induced force between two identical uncharged spheres
}

B. J. Cox

University of Wollongong, barryc@uow.edu.au

N. Thamwattana

University of Wollongong, ngamta@uow.edu.au

James M. Hill

University of Wollongong, jhill@uow.edu.au

Follow this and additional works at: https://ro.uow.edu.au/infopapers

Part of the Physical Sciences and Mathematics Commons

\section{Recommended Citation}

Cox, B. J.; Thamwattana, N.; and Hill, James M.: Electric field-induced force between two identical uncharged spheres 2006.

https://ro.uow.edu.au/infopapers/337

Research Online is the open access institutional repository for the University of Wollongong. For further information contact the UOW Library: research-pubs@uow.edu.au 


\title{
Electric field-induced force between two identical uncharged spheres
}

\begin{abstract}
The problem of electric field-induced force between spheres is fundamental to electrorheological fluids. Previously published experimental results indicate that the interaction force between two spheres under an external field is not adequately explained by the existing approximate and numerical methods. The specific models compared were dipole, dipole with local field corrections and a finite-element analysis. This letter employs an exact solution (via the equivalent multipole-moment method) to the electrostatic problem which accurately predicts the low-frequency experimental results at all measured interstices. The solution presented later is self-contained and addresses specifically the geometry of the previously mentioned experimental results. While more general solutions have been published previously, they are more complex than is required by this problem. The solution presented here is accurate for all sphere spacings, but in particular could apply to nano-spheres in close proximity.

\section{Disciplines}

Physical Sciences and Mathematics

Publication Details

This article was originally published as Cox, BJ, Thamwattana, N \& Hill, JM, Electric field-induced force between two identical uncharged spheres, Applied Physics Letters, 2006, 88, 152903. Original journal available here.
\end{abstract}




\title{
Electric field-induced force between two identical uncharged spheres
}

\author{
Barry J. Cox, ${ }^{a}$ Ngamta Thamwattana, and James M. Hill \\ Nanomechanics Group, School of Mathematics and Applied Statistics, University of Wollongong, \\ Wollongong, NSW, 2522, Australia
}

(Received 27 October 2005; accepted 17 February 2006; published online 10 April 2006)

\begin{abstract}
The problem of electric field-induced force between spheres is fundamental to electrorheological fluids. Previously published experimental results indicate that the interaction force between two spheres under an external field is not adequately explained by the existing approximate and numerical methods. The specific models compared were dipole, dipole with local field corrections and a finite-element analysis. This letter employs an exact solution (via the equivalent multipole-moment method) to the electrostatic problem which accurately predicts the low-frequency experimental results at all measured interstices. The solution presented later is self-contained and addresses specifically the geometry of the previously mentioned experimental results. While more general solutions have been published previously, they are more complex than is required by this problem. The solution presented here is accurate for all sphere spacings, but in particular could apply to nano-spheres in close proximity. (C) 2006 American Institute of Physics.
\end{abstract}

[DOI: 10.1063/1.2185607]

It is well known that a dielectric body subject to an external electric field becomes polarized with bound charge, and when two bodies are present they interact producing a perturbation to these bound charges. Also, when the bodies are appropriately aligned, an electrostatic force will be generated between the two bodies. As described in Wang, Peng, $\mathrm{Lu}$ and $\mathrm{Wen}^{1}$ an experiment was conducted to measure the field-induced interaction force between two spheres at small interstices. The procedure of interest was the placing of two $6.3 \mathrm{~mm}$ diameter spheres of $\mathrm{SrTiO}_{3}$ at interstices varying from 0.01 to $0.8 \mathrm{~mm}$ and subject to electric fields of varying strength and measuring the mutual force of attraction. This experiment confirmed that there is a quadratic relationship between applied field strength and force of attraction at all interstices, as predicted by electrostatic dipole theory. However, the result of interest is that at small interstices the force of attraction is much greater than that predicted by the theoretical models employed in that paper. It should be noted that the theoretical approximations were not all intended to be employed for small interstices, and therefore, to a certain extent, some disagreement was expected.

The first theoretical model used in Ref. 1 was the dipole model as described by Coulson. ${ }^{2}$ This model is based on calculating the force of attraction by considering the two spheres as ideal point dipoles. This has the effect of ignoring any interaction between the induced bound charge of one sphere with the other, as well as not correctly accounting for the bound charge distribution over the surface of the spheres. Both of these phenomena are expected to be more significant at small interstices and so it is not surprising that this method did not provide an accurate prediction of the results. The second theoretical model employed in the comparison was an improvement on the dipole model which applied a correction for local field effects as presented by Davis. ${ }^{3}$ While this model begins to provide an account of the interaction between the spheres it only goes to one level of interaction. As recognized by the theory of infinite images, each induced charge in the first sphere will perturb the bound charge of the

\footnotetext{
${ }^{\text {a) }}$ Author to whom correspondence should be addressed; electronic mail:
} bjc949@uow.edu.au second sphere and that perturbation will in turn interact with and perturb the bound charge of the first sphere. Again, at very small interstices, this phenomenon becomes significant and limits the use of this method for such situations. Finally, a finite-element approach as described by Davis ${ }^{4}$ was also included in the comparison. This method provided by far the most qualitative agreement with the experimental results. However, even in this case Wang et al. ${ }^{1}$ reported that "it should be emphasized that all three models are no longer valid for the case of a very thin gap" and "this result indicates that these available calculations for the mutual forces should be improved when the spheres are arranged closely."

The method of equivalent multipole moments around an axis of symmetry is a known technique which can be applied to this and similar problems. This method was first proposed by Washizu, ${ }^{5}$ developed by Matsuyama, Yamamoto and Washizu $^{6}$ and Washizu and Jones ${ }^{7}$ present a solution for two identical spheres in an arbitrarily aligned external field. An advantage of this technique is that effectively Laplace's equation is solved around a number of origins allowing the application of simple and axially independent spherical coordinate systems at each origin. The reexpansion formula then allows a simple expansion around one origin to be re-expanded around any other point lying on the axis of symmetry.

In this letter the solution developed by Washizu and Jones $^{7}$ is applied to the specific case of two homogeneous dielectric spherical particles subject to an external electric field applied in a direction parallel to the line of centers of the two particles. This external field induces a bound charge of polarization in each particle which then interact with each other inducing further polarization. It is demonstrated that this phenomenon is particularly significant when the particles are in close proximity relative to the particle radius. A calculation of the electrostatic force experienced by the particles is determined and shown to agree closely with the experimental results reported in Ref. 1. Details of the solution will be presented later.

The numerical results were derived by solving the matrix equation given later for a suitably large matrix. Once the coefficients of the potential expansion were determined they 


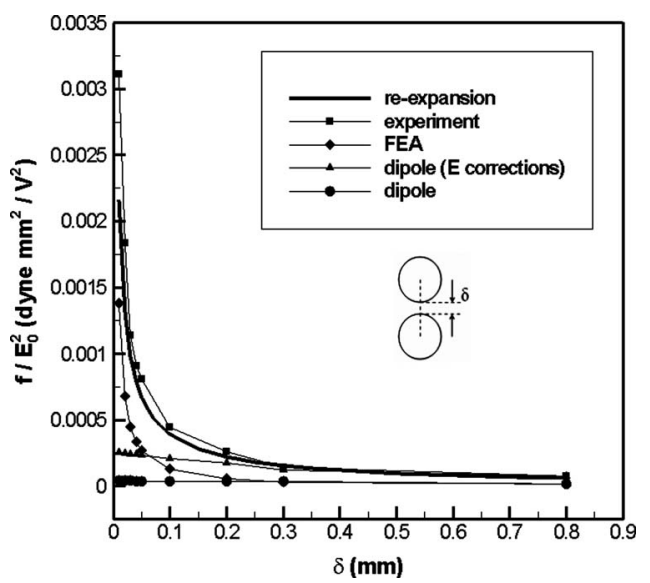

FIG. 1. Comparison between the results original reported by Wang et al. (see Ref. 1) and the present exact solution.

were then applied to the formula for the force (16) which can be directly compared to the experimental results of Wang et $a l .{ }^{1} \quad$ The calculations were performed employing a $100 \times 100$ matrix which in all cases led to a final term which was at least 1000 times smaller than the first term. The calculations were repeated with the four times larger $200 \times 200$ matrix and a difference was only discernible for interstices less than $0.05 \mathrm{~mm}$. Predictably the highest relative error occurred with the lowest interstice of $0.01 \mathrm{~mm}$. However, even here the relative error between the $100 \times 100$ and the $200 \times 200$ result was only 0.000128 . The results from the present solution are presented in Fig. 1 together with the experimental values and the previously reported values for the three approximation methods considered in the original letter.

The results of the Wang et al. ${ }^{1}$ are in a mixture of units, however the systeme International system of units was assumed for the solution presented here. Therefore, to assist in the comparison the results given here are subsequently converted back to the units of Ref. 1 . The specific values used were $a=3.15 \mathrm{~mm}, d=\delta+6.3 \mathrm{~mm}$, where $\delta$ varies from 0.8 to $0.01 \mathrm{~mm}$ and $k=294$. As the results were normalized to $F_{e} / E^{2}$ the applied field strength of $1 \mathrm{~V} \mathrm{~m}^{-1}$ was used. However, the resulting force of attraction is then multiplied by a factor of $10^{11}$ to return it to the units used by Wang et al., ${ }^{1}$ that is dyne $\mathrm{mm}^{2} \mathrm{~V}^{-2}$.

As can be seen from Fig. 1, the predictions from the present reexpansion method achieve a high level of agreement with the experimental results. Although at low interstices the agreement seems to be not quite as strong, it should be noted that the gradient of the graph in this region is such that any measurement error in the size of the interstice is likely to have a significant impact on the measurement of the mutual force of attraction. In any case, the predictions of the reexpansion method are a significant improvement on the three calculations which were used in the original comparison. It is for these reasons that the authors believe that the present reexpansion method for calculation of mutual forces between dielectric particles in electric fields provides the accuracy required by the problem and addresses the concerns raised from the experiment conducted by Wang et al. ${ }^{1}$

Two identical spherical particles (of radius $a$ and dielectric constant $k$ ), $A$ and $B$, are in a vacuum with their centers a distance $d$ apart, where $d=2 a+\delta$, where $\delta \geqslant 0$. We define a Downloaded 14 Aug 2006 to 130.130 .37 .6 . Redistribution subject
Cartesian coordinate system $(x, y, z)$ with the origin at the midpoint of the centers of the particles, and aligned so that the $z$ axis passes through the centers of the particles in the direction from $A$ to $B$. We also define two separate axially symmetric spherical coordinate systems, $(r, \theta)$ and $(R, \Theta)$ with their origins located at the center of particles $A$ and $B$, respectively, and with the angular variables $\theta$ and $\Theta$ measured from the positive direction of $z$. An external electric field of strength $E$ is applied in the positive $z$ direction, and the whole configuration is illustrated in Fig. 2.

The scalar electrostatic potentials are governed by Laplace's equation, $\nabla^{2} \phi=0$. In the vacuum external to both particles there is the potential of the applied electric field, $-E z$, and a perturbation created by the polarization of each particle which we express as an expansion centered at the respective particles. Since this perturbation must vanish at infinity, the non-negative radial powers can be immediately neglected. This leads us to the following uniformly convergent expansion for the external potential

$$
\phi_{o}=\sum_{n=0}^{\infty}\left[a_{n}\left(\frac{a}{r}\right)^{n+1} \mathbf{P}_{n}(\lambda)+A_{n}\left(\frac{a}{R}\right)^{n+1} \mathbf{P}_{n}(\Lambda)\right]-E z,
$$

where $\lambda=\cos \theta, \Lambda=\cos \Theta$ and $\mathbf{P}_{n}$ is the usual Legendre polynomial of order $n$. Inside the spheres, the expansion of the potential will not include the negative radial powers, since they are singular at the respective origins. That is

$$
\phi_{i}=\sum_{n=0}^{\infty} c_{n}\left(\frac{r}{a}\right)^{n} \mathbf{P}_{n}(\lambda), \quad \Phi_{i}=\sum_{n=0}^{\infty} C_{n}\left(\frac{R}{a}\right)^{n} \mathbf{P}_{n}(\Lambda),
$$

where $\phi_{i}$ and $\Phi_{i}$ are the potentials internal to particles $A$ and $B$, respectively.

As suggested by Washizu, ${ }^{5}$ the terms of the Legendre expansion of an axially symmetric potential in one spherical coordinate system can be reexpanded at another point on the axis of symmetry. In particular

$$
\left(\frac{a}{R}\right)^{n+1} \mathbf{P}_{n}(\Lambda)=(-1)^{n}\left(\frac{a}{d}\right)^{n+1} \sum_{m=0}^{\infty} \frac{(m+n) !}{m ! n !}\left(\frac{r}{d}\right)^{m} \mathbf{P}_{m}(\lambda) .
$$

A proof of this formula is given in Appendix of Matsuyama, Yamamoto, and Washizu, ${ }^{6}$ and it is important to note that this expansion is only convergent in the domain $r<d$, and that in our coordinate systems $z=r \cos \theta-d / 2$. Therefore, we can express Eq. (1) in the first spherical coordinate system as

$$
\begin{gathered}
\phi_{o}=\sum_{n=0}^{\infty}\left[a_{n}\left(\frac{a}{r}\right)^{n+1}+b_{n}\left(\frac{r}{a}\right)^{n}\right] \mathbf{P}_{n}(\lambda)-E r \mathbf{P}_{1}(\lambda)+\frac{E d}{2} \mathbf{P}_{0}(\lambda), \\
b_{n}=-\left(\frac{a}{d}\right)^{n} \sum_{m=0}^{\infty}\left(-\frac{a}{d}\right)^{m+1} \frac{(m+n) !}{m ! n !} A_{m},
\end{gathered}
$$

and we note that $\mathbf{P}_{0}(\lambda)=1$, and $\mathbf{P}_{1}(\lambda)=\lambda$. Symmetry allows us to rewrite the $b_{n}$ in terms of $a_{n}$ instead of $A_{n}$,

$$
b_{n}=-\left(\frac{a}{d}\right)^{n} \sum_{m=0}^{\infty}\left(\frac{a}{d}\right)^{m+1} \frac{(m+n) !}{m ! n !} a_{m},
$$

and, in particular, when $n=0, b_{0}=-\sum_{m=0}^{\infty}(a / d)^{m+1} a_{m}$.

From this point onwards we will only be concerned with developing a solution for sphere $A$. Once $a_{n}$ has been determined, and using the expressions above, the solution for sphere $B$ will follow immediately. 


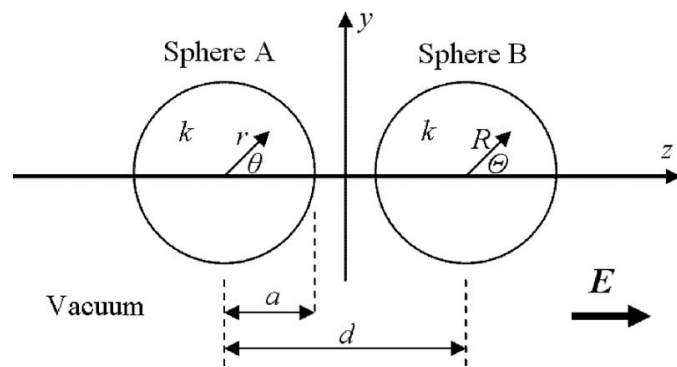

FIG. 2. Problem geometry showing particles and two spherical polar coordinate systems $(r, \theta)$ and $(R, \Theta)$.

The electrostatic potential continuity boundary conditions apply at the surfaces of the spheres. Namely, $\phi_{i}=\phi_{o}$, and $k \partial \phi_{i} / \partial r=\partial \phi_{o} / \partial r$, at $r=a$. These boundary conditions leads to $a_{1}=\left(E a-b_{1}\right)(k-1) /(k+2)$, and for $n>1$

$$
a_{n}=\frac{n(k-1)}{n(k+1)+1}\left(\frac{a}{d}\right)^{n} \sum_{m=0}^{\infty}\left(\frac{a}{d}\right)^{m+1} \frac{(m+n) !}{m ! n !} a_{m} .
$$

We can also use a simultaneous solution of the equations above to deduce $c_{n}=-a_{n}(2 n+1) /[n(k-1)]$ for $n>0$.

These equations can now be expressed in matrix form in the following way. First we define $\mathbf{A}$ and $\mathbf{V}$ as column vectors with elements $\left[a_{1}, a_{2}, \ldots, a_{N}\right]$ and $[E a(k-1) /(k$ $+2), 0, \ldots, 0]$, respectively, and $\mathbf{U}$ is a square matrix whose elements are defined by

$$
U_{n, m}=\frac{n(k-1)}{n(k+1)+1}\left(\frac{a}{d}\right)^{m+n+1} \frac{(m+n) !}{m ! n !} .
$$

Then we can express the equations for $a_{1}$ and $a_{n}$ (7), in the single matrix equation $\mathbf{A}=\mathbf{U A}+\mathbf{V}$. This can be easily solved for $\mathbf{A}$ as $\mathbf{A}=(\mathbf{I}-\mathbf{U})^{-1} \mathbf{V}$, where $\mathbf{I}$ is the $N \times N$ identity matrix. We note that this is equivalent to Eq. (3.16) in Ref. 7.

The values of $a_{n}$, once determined, can be applied in the potential expansion (4), remembering that $A_{n}$ is already determined in terms of $a_{n}$ via the symmetry relationship. So the potential everywhere outside both the spheres is given by

$$
\phi_{o}=\sum_{n=1}^{\infty} a_{n}\left[\left(\frac{a}{r}\right)^{n+1} \mathbf{P}_{n}(\lambda)+\left(-\frac{a}{R}\right)^{n+1} \mathbf{P}_{n}(\Lambda)\right]-E z,
$$

for $r, R \geqslant a$, and in the vicinity of sphere $A$

$\phi_{o}=\frac{E d}{2}+\sum_{n=1}^{\infty} a_{n}\left\{\left[\left(\frac{a}{r}\right)^{n+1}-\frac{n(k+1)+1}{n(k-1)}\left(\frac{r}{a}\right)^{n}\right] \mathbf{P}_{n}(\lambda)-\left(\frac{a}{d}\right)^{n+1}\right\}$,

for $a \leqslant r<d$. Inside of sphere $A$ the potential as defined by Eq. $(2)_{1}$ after applying the above results gives the:

$$
\phi_{i}=\frac{E d}{2}-\sum_{n=1}^{\infty} a_{n}\left[\left(\frac{a}{d}\right)^{n+1}+\frac{2 n+1}{n(k-1)}\left(\frac{r}{a}\right)^{n} \mathbf{P}_{n}(\lambda)\right],
$$

for $r \leqslant a$. The symmetry of the solution also allows an immediate expression of the potential inside of sphere $B$ as

$$
\Phi_{i}=-\frac{E d}{2}+\sum_{n=1}^{\infty} a_{n}\left[\left(\frac{a}{d}\right)^{n+1}+\frac{2 n+1}{n(k-1)}\left(-\frac{R}{a}\right)^{n} \mathbf{P}_{n}(\Lambda)\right] \text {. }
$$

Following along the lines of Washizu and Jones ${ }^{7}$ the electrostatic force is calculated from Coulomb's law, and the first step is to calculate the notional surface charge density of sphere $A$. In our case, this charge is solely the bound (polar- ization) charge since the spheres themselves carry no free charge. Applying Gauss's flux theorem in a vacuum to a very thin volume enclosing a small portion of the surface of sphere $A$ gives $\rho=\epsilon_{0}\left(\partial \phi_{i} / \partial r-\partial \phi_{o} / \partial r\right)_{r=a}$, where $\rho$ is the surface charge density and $\epsilon_{0}$ is the permittivity of free space. Applying the solutions for the potential [(10) and (11)] gives $\rho=\left(\epsilon_{0} / a\right) \sum_{n=1}^{\infty}(2 n+1) a_{n} \mathbf{P}_{n}(\lambda)$.

Now the electrostatic force is the interaction of this surface charge with the electrostatic field outside of the sphere which is not a result of this surface charge. That is, the potential to be used is that from Eq. (10) but excluding the terms resulting from the polarization of sphere $A$. This potential is

$$
\phi_{o}^{\star}=\frac{E d}{2}-\sum_{n=1}^{\infty} a_{n}\left[\frac{n(k+1)+1}{n(k-1)}\left(\frac{r}{a}\right)^{n} \mathbf{P}_{n}(\lambda)+\left(\frac{a}{d}\right)^{n+1}\right] .
$$

Since the solution is symmetric around the $z$ axis, only the $z$ components of the field are required for the calculation. Remembering that $\mathbf{E}=-\nabla \phi_{o}^{\star}$, then $E_{z}=-\partial \phi_{o}^{\star} / \partial z$, at $r=a$, where $E_{z}$ is the $z$ component of the electrostatic force on the surface of sphere $A$. Using $\partial / \partial z=\cos \theta \partial / \partial r-(\sin \theta / r) \partial / \partial \theta$ to perform this differentiation and applying the value of $r=a$ yields

$$
\begin{aligned}
E_{z}= & \frac{1}{a(k-1)} \sum_{n=1}^{\infty} \frac{n(k+1)+1}{n} a_{n}\left[\left(1-\lambda^{2}\right) \mathbf{P}_{n}^{\prime}(\lambda)\right. \\
& \left.+n \lambda \mathbf{P}_{n}(\lambda)\right] .
\end{aligned}
$$

From the well-known identity of the Legendre polynomials $\left(1-x^{2}\right) \mathbf{P}_{n}^{\prime}(x)+n x \mathbf{P}_{n}(x)=n \mathbf{P}_{n-1}(x)$, this simplifies to

$$
E_{z}=\frac{1}{a(k-1)} \sum_{n=0}^{\infty}[(n+1)(k+1)+1] a_{n+1} \mathbf{P}_{n}(\lambda) .
$$

With these two results determined, the force is calculated via the following integral, $F_{e}=\int_{0}^{\pi} E_{z} \rho 2 \pi a^{2} \sin \theta d \theta$. Substituting for $E_{z}$ and $\rho$ and applying the orthogonality condition for Legendre polynomials yields

$$
F_{e}=\frac{4 \pi \epsilon_{0}}{k-1} \sum_{n=1}^{\infty}[(n+1)(k+1)+1] a_{n} a_{n+1} .
$$

Note that in this case a positive value for $F_{e}$ indicates a force in the positive $z$ direction. That is, a force of attraction towards sphere $B$. We comment that this equation is equivalent to Eq. (4.12) in Ref. 7 and the second term in the solution developed in Ref. 8.

The support of the Australian Research Council through the Discovery Project Grant Scheme is gratefully acknowledged. The authors are grateful to Professor Weijia Wen for drawing their attention to existing deficiencies in solution procedures for this problem.

${ }^{1}$ Z. Wang, Z. Peng, K. Lu, and W. Wen, Appl. Phys. Lett. 82, 1796 (2003).

${ }^{2}$ C. A. Coulson, Electricity, 5th ed. (Oliver \& Boyd, Edinburgh, 1958).

${ }^{3}$ L. C. Davis, J. Appl. Phys. 72, 1334 (1992).

${ }^{4}$ L. C. Davis, Appl. Phys. Lett. 60, 319 (1992).

${ }^{5}$ M. Washizu, J. Electrost. 29, 177 (1992).

${ }^{6}$ T. Matsuyama, H. Yamamoto, and M. Washizu, J. Electrost. 36, 195 (1995).

${ }^{7}$ M. Washizu and T. B. Jones, IEEE Trans. Ind. Appl. 32, 233 (1996).

${ }^{8}$ Y. Nakajima and T. Sato, J. Electrost. 45, 213 (1999). 